\title{
Light-modulated vertical heterojunction phototransistors with distinct logical photocurrents
}

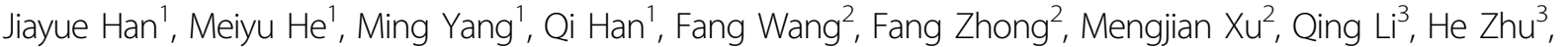 \\ Chongxin Shan ${ }^{4}$, Weida Hu $\mathbb{D}^{2,3}$, Xiaoqing Chen ${ }^{5}$, Xinran Wang $\mathbb{B}^{5}$, Jun Gou ${ }^{1,6}$, Zhiming Wu $\mathbf{u}^{1,6}$ and Jun Wang $\mathbb{B}^{1,6}$
}

\begin{abstract}
The intriguing carrier dynamics in graphene heterojunctions have stimulated great interest in modulating the optoelectronic features to realize high-performance photodetectors. However, for most phototransistors, the photoresponse characteristics are modulated with an electrical gate or a static field. In this paper, we demonstrate a graphene/ $C_{60} /$ pentacene vertical phototransistor to tune both the photoresponse time and photocurrent based on light modulation. By exploiting the power-dependent multiple states of the photocurrent, remarkable logical photocurrent switching under infrared light modulation occurs in a thick $C_{60}$ layer $(11 \mathrm{~nm})$ device, which implies competition of the photogenerated carriers between graphene/ $C_{60}$ and $C_{60} /$ pentacene. Meanwhile, we observe a complete positive-negative alternating process under continuous $405 \mathrm{~nm}$ irradiation. Furthermore, infrared light modulation of a thin $C_{60}(5 \mathrm{~nm})$ device results in a photoresponsivity improvement from $3425 \mathrm{~A} / \mathrm{W}$ up to $7673 \mathrm{~A} / \mathrm{W}$, and we clearly probe the primary reason for the distinct modulation results between the 5 and $11 \mathrm{~nm} \mathrm{C}_{60}$ devices. In addition, the tuneable bandwidth of the infrared response from 10 to $3 \times 10^{3} \mathrm{~Hz}$ under visible light modulation is explored. Such distinct types of optical modulation phenomena and logical photocurrent inversion characteristics pave the way for future tuneable logical photocurrent switching devices and high-performance phototransistors with vertical graphene heterojunction structures.
\end{abstract}

\section{Introduction}

Graphene has promising potential in fabricating phototransistors and photodetectors owing to its unique band structure with prominent optoelectronic and charge transport properties ${ }^{1-3}$. Tremendous prospects have emerged in transparent devices ${ }^{4,5}$, silicon compatible devices $^{6}$, and optical modulators ${ }^{7-9}$. However, the inferior light absorption of single atomic layer graphene limits the

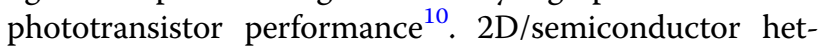
erojunctions are expected to break the electron-hole occupation symmetry, giving rise to the generation of photocurrents, including in quantum dot-enhanced

Correspondence: Weida Hu (wdhu@mail.sitp.ac.cn) or

Xinran Wang (xrwang@nju.edu.cn) or Jun Wang (wjun@uestc.edu.cn)

${ }^{1}$ School of Optoelectronic Science and Engineering, University of Electronic

Science and Technology of China, Chengdu 610054, China

${ }^{2}$ State Key Laboratory of Infrared Physics, Shanghai Institute of Technical

Physics, Chinese Academy of Science, 500 Yutian Road, Shanghai 200083,

China

Full list of author information is available at the end of the article structures $^{11,12}$, stacked 2D heterojunction structures ${ }^{13-17}$, 2D planar structures ${ }^{18,19}$, dual photogating phototransistors ${ }^{20}$, waveguide-integrated enhanced structures $^{21,22}$, bilayer-enhanced structures ${ }^{23,24}$, and even nanographite structures ${ }^{25,26}$. In particular, some typical 2D localized field methods incorporating 2D, organic, perovskite, and Dirac materials were subsequently proposed to realize high-performance phototransistors ${ }^{23,24,27-33}$. Numerous localized field-enhanced phototransistors have been integrated with an electrostatic field to achieve distinct features such as a floating-gate structure ${ }^{27,28}$, a builtin field ${ }^{19}$, a photogating electric field ${ }^{23,24,29,30}$ and a ferroelectric field ${ }^{31}$. For example, the ferroelectric field aims to suppress the dark current, and the floating gate aims to promote the light sensitivity.

As another unprecedented external intervention, light modulation is seldom researched for tuning optoelectronic properties. A method involving $450 \mathrm{~nm}$ light illumination that can expand the photodetection range and

\section{(c) The Author(s) 2020}

(c) (i) Open Access This article is licensed under a Creative Commons Attribution 4.0 International License, which permits use, sharing, adaptation, distribution and reproduction cc) in any medium or format, as long as you give appropriate credit to the original author(s) and the source, provide a link to the Creative Commons license, and indicate if changes were made. The images or other third party material in this article are included in the article's Creative Commons license, unless indicated otherwise in a credit line to the material. If material is not included in the article's Creative Commons license and your intended use is not permitted by statutory regulation or exceeds the permitted use, you will need to obtain permission directly from the copyright holder. To view a copy of this license, visit http://creativecommons.org/licenses/by/4.0/. 


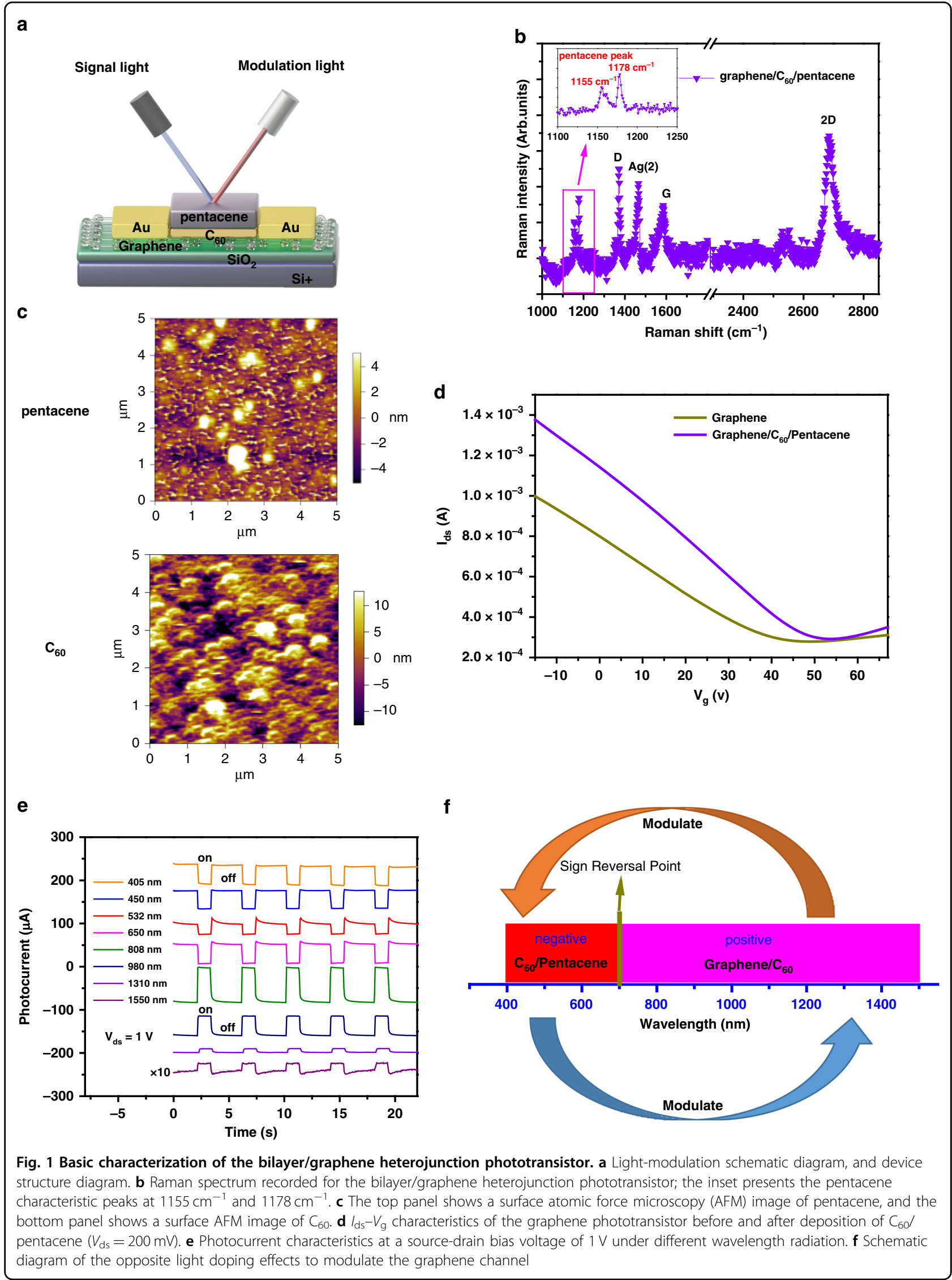


suppress the dark current was proposed by Fang et al. ${ }^{34}$. Furthermore, Gao et al. ${ }^{35}$ demonstrated Schottky phototransistors with enhanced photocurrents due to a Schottky-Ohmic transformation induced by light. As we known, comprehensive modulation of the photocurrent magnitude, operating speed, and signal direction of phototransistors by modulation light input is lacking, where light modulation hopefully offers a versatile platform for replacing electrical modulation to explore more intriguing optoelectronic devices.

In this paper, we demonstrate a light modulation method to adjust the response magnitude, speed, and direction of photocurrents based on graphene/ $C_{60} /$ pentacene phototransistors with different thickness $\mathrm{C}_{60}$. We observe a logical photocurrent reversal and photocurrentresponse time dynamics (response time varies from 550 to $20 \mu \mathrm{s})$ driven by light modulation in a thick-intermediatetransport layer device. In addition, in the thin $\mathrm{C}_{60}(5 \mathrm{~nm})$ phototransistor, we exploit an efficient method to provide a nearly two- to threefold improvement in the visible light photoresponsivity and up to a $10^{3}$-fold improvement in the infrared response time under suitable light modulation. In addition, we observe both positive and negative alternating photocurrents as a function of the incident light power density. This peculiar phenomenon enables us to analyze the charge-transfer $(\mathrm{CT})$ process and study the potential for new functional devices in the graphene $/ \mathrm{C}_{60} /$ pentacene system. The combination of distinct mechanisms and novel light modulation extends the functions of conventional phototransistors and promotes the device performance.

\section{Results}

The light modulation transistor based on the vertical graphene/ $\mathrm{C}_{60}$ /pentacene heterojunction in this study has a typical photoconductive structure, with the schematic depicted in Fig. 1a. The bilayer heterojunction $\mathrm{C}_{60} /$ pentacene acts as the main light-absorbing layer, which allows photogenerated free carriers to be injected into graphene (single-layer graphene is confirmed by Raman spectroscopy, Fig. 1b), modulating the conductance of the channel. $\mathrm{C}_{60}$ /pentacene bilayer small molecule films with 5 and $10 \mathrm{~nm}$ thicknesses are deposited onto monolayer graphene with previously prepared electrodes (see Materials and methods in the supporting information for details). The characteristic peaks of $10 \mathrm{~nm}$ pentacene emerge exclusively at 1155 and $1178 \mathrm{~cm}^{-1}$, whereas $5 \mathrm{~nm}$ $\mathrm{C}_{60}$ is characterized by the $\mathrm{A}_{\mathrm{g}}(2)$ peak $\left(1464 \mathrm{~cm}^{-1}\right)$. Valid interface contact and a smooth surface will ensure the carrier transport efficiency of the device. Therefore, morphological characterization of pentacene by atomic force microscopy was performed, showing a relatively flat surface in Fig. 1c (top panel), and the intermediate layer $\mathrm{C}_{60}$ is characterized in Fig. 1c (bottom panel). The $\mathrm{C}_{60} /$ graphene contact breaks the electron-hole occupation symmetry in intrinsic graphene and tunes the Fermi level of graphene. Owing to the $\mathrm{C}_{60}$ /graphene contact interaction, we estimate that the Dirac point of graphene shift toward positive voltages, indicating hole injection from $\mathrm{C}_{60}$ to graphene (Fig. 1d).

Because of the limited absorption range of $\mathrm{C}_{60}$ /pentacene with a cutoff point of $\sim 700 \mathrm{~nm}$ (Fig. S1), the prepared phototransistor shows positive and negative alternating photocurrents under different wavelength (from 405 to $1550 \mathrm{~nm}$ ) illumination while keeping $V_{\text {sd }}$ and the power density constant (Fig. 1e). The negative photocurrents stem from $\mathrm{C}_{60}$ /pentacene electron injection into graphene, and the graphene $/ C_{60}$ heterojunction determines the positive response ${ }^{23}$. The positive photocurrents in the infrared region and negative photocurrents in the visible region suggest opposite light doping effects that modulate the graphene channel. The distinct optical doping effects are utilized to realize inhibition or promotion of the built-in field in the vertical heterojunction, and we use them to modulate the original optical signal. (Fig. 1a, f, opposite response regions act as signal light and modulation light).

Contrary to the conventional scenario (in which positive and negative responses will inhibit each other), a peculiar situation occurs in our measurements. We excited a device with pulsed visible signal light (power density of $20 \mathrm{~mW} / \mathrm{cm}^{2}$ ) by infrared optical on-off modulation, as shown in Fig. 2a. The optically modulated output signal current significantly rises nearly two- to threefold under $20 \mathrm{~mW} / \mathrm{cm}^{2}$ infrared light and stably recovers after the modulation light is removed. In addition, different powers of the signal light or modulation light affect the trend of the output current. Figure $2 \mathrm{~b}$ depicts the modulated dynamics of the output currents under various light power densities; $\beta$ ( $\beta$ is an enhancement factor for easy comparison, which replaces modulated $I_{\mathrm{ph}} /$ signal $I_{\mathrm{ph}}$ ) decreases sharply as the modulation light power density increases at relatively low signal light power densities (251.7 and $55.7 \mu \mathrm{W} / \mathrm{cm}^{2}$ ), while there is a slight rise in $\beta$ at higher signal light power densities. As the bandgap of bilayer $\mathrm{C}_{60}$ /pentacene forms a type-II heterojunction, the N-type $\mathrm{C}_{60}$ with a LUMO (HOMO) of $4.5 \mathrm{eV}(6.2 \mathrm{eV})$ and P-type pentacene with a value of $3.0 \mathrm{eV}(4.9 \mathrm{eV})$ (Fig. 2c) ${ }^{36-38}$ enable photogenerated electrons to propagate into graphene. The tuneable Schottky barrier derived from graphene $/ \mathrm{C}_{60}$ affects the band alignment. The Fermi level of the bottom graphene is closely associated with the modulation light power density, which conclusively inhibits or facilitates carrier transfer. Therefore, the infrared modulation light causes P-type doping in graphene and directly enhances the built-in field of $\mathrm{C}_{60}$ /pentacene (Fig. 2c), which allows electrons (signal light generated) in $\mathrm{C}_{60}$ /pentacene to effectively be injected into graphene, 


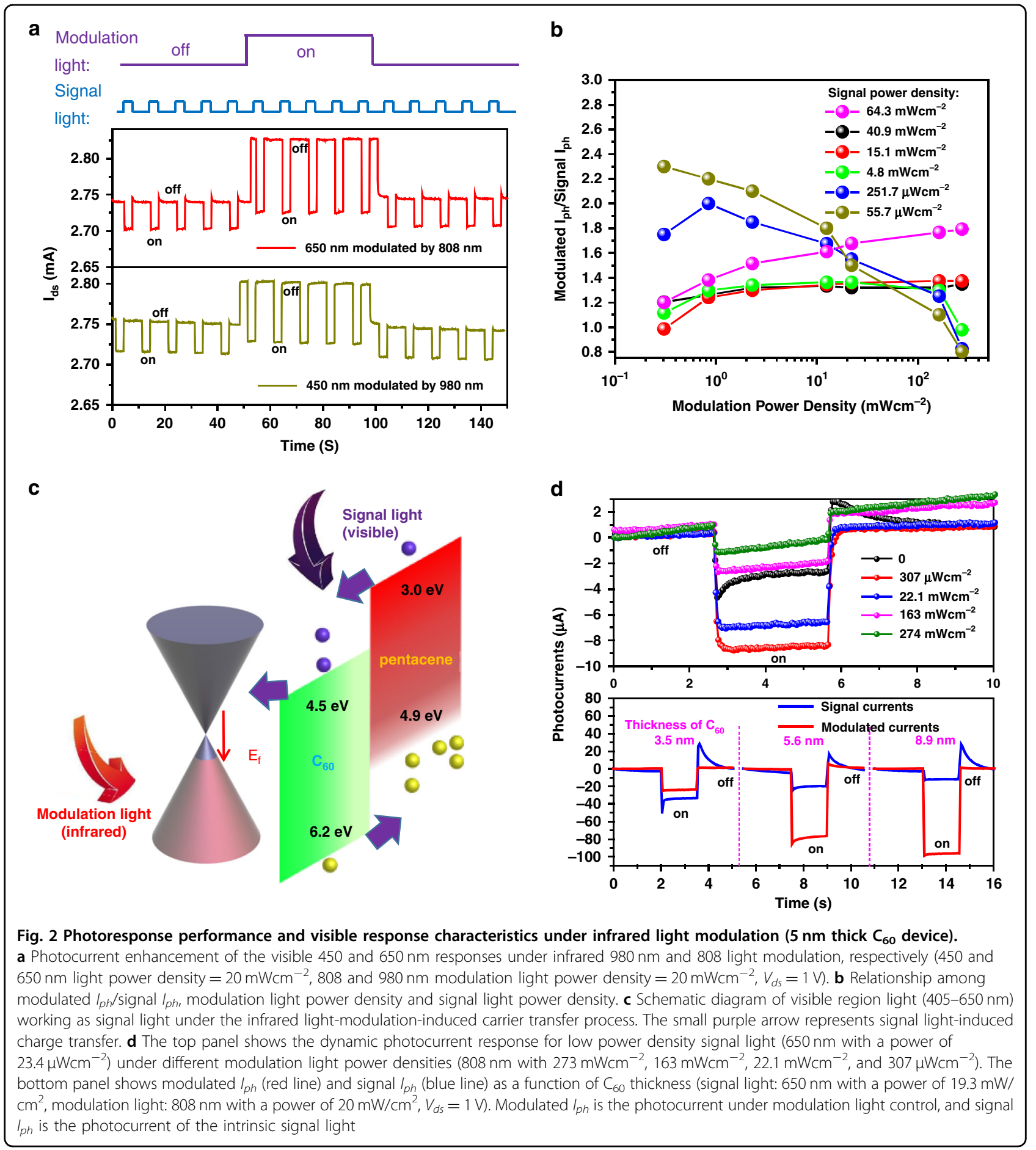

resulting in a higher photocurrent (Fig. 2a). The appropriate power of the infrared light will cause P-doping of graphene and enable the signal light photogenerated electrons to be injected into graphene. However, excessive power of the infrared light can cause deep P-doping of graphene. Then, the signal light photogenerated electrons hardly induce a variation in the conductance due to the considerable quantity of carriers with the deep P-doping of graphene (Fig. 2d top panel) ${ }^{39}$. Therefore, matching the powers of the modulation light and signal light will lead to a larger enhancement (Fig. 2b).

By utilizing this trend, the photoresponsivity of the intrinsic visible signal light $\left(15.4 \mu \mathrm{W} / \mathrm{cm}^{2}\right)$ can be promoted from $3425 \mathrm{~A} / \mathrm{W}$ to $7673 \mathrm{~A} / \mathrm{W}$ under a proper 
modulation light power density of $307 \mu \mathrm{W} / \mathrm{cm}^{2}$ (Fig. $2 \mathrm{~d}$ top panel). Under the same signal $(650 \mathrm{~nm}$ with $19.3 \mathrm{~mW} /$ $\left.\mathrm{cm}^{2}\right)$ and modulation $\left(808 \mathrm{~nm}\right.$ with $\left.22.1 \mathrm{~mW} / \mathrm{cm}^{2}\right)$ light, we examined the different thickness $\mathrm{C}_{60}$ devices (Fig. $2 \mathrm{~d}$ bottom panel). As the thickness of $\mathrm{C}_{60}$ increases, $\beta$ can reach 7.92, and a further explanation of the thickness variation effect will be introduced below. The device modulation performance also depends on the light modulation response, such as the inferior response when $1550 \mathrm{~nm}$ light acts as the modulation light, which has a relatively weak ability to realize enhanced modulation (Fig. S2).

To deeply clarify the light modulation mechanism mentioned above, we turn our focus to the thicknessdependent modulation in graphene $/ \mathrm{C}_{60} /$ pentacene. We fabricate an $11 \mathrm{~nm} \mathrm{C}_{60}$ control device with the same pentacene thickness, which aims to force photogenerated electrons to be partly depleted at a distance (the effective exciton length is normally $5-10 \mathrm{~nm})^{39}$. Compared with the previous $5 \mathrm{~nm}$ thick $\mathrm{C}_{60}$ light modulation measurements, which can only realize codirectional photocurrent modulation (Fig. 2a), the phototransistor with $650 \mathrm{~nm}$ signal light demonstrates obvious photocurrent polarity switching when modulated by infrared 808 or $980 \mathrm{~nm}$ light, as shown in Fig. 3a. This logical signal reversal by light modulation exhibits some similar features to optical inversion devices ${ }^{40-43}$. When the thickness of the intermediate transport layer exceeds or approaches the effective built-in electric field length, the entire system can be split into two parts, as displayed in Fig. 3b, c (the red dashed line in Fig. 3b): graphene/ $\mathrm{C}_{60}-1$ and $\mathrm{C}_{60}-2$ /pentacene. The $\mathrm{C}_{60}-2$ /pentacene will exhibit a response for a radiation change of $400-700 \mathrm{~nm}$, with its bandgap approaching $2 \mathrm{eV}$, and prompt electron movement toward graphene $/ \mathrm{C}_{60}-1$ (as we note in Fig. $3 \mathrm{c}$ with an arrow). Owing to the formation of a CT state at the graphene/ $\mathrm{C}_{60}-1$ interface (near-infrared region), appropriate energy photogenerated electrons can transfer into shallow impurities of $\mathrm{C}_{60}$ and be trapped in them. Photogenerated holes from $\mathrm{C}_{60}$ move into graphene under visible illumination. Therefore, both near-infrared and visible irradiation will produce positive photocurrents at the graphene/ $\mathrm{C}_{60}-1$ interface, suggesting that the transmission direction of electrons points from graphene to $\mathrm{C}_{60}$ (as depicted in Fig. $3 \mathrm{c}$ with an arrow). Thus, if visible light irradiates the graphene $/ \mathrm{C}_{60}$ /pentacene heterojunction, then the opposite transfer directions derived from graphene $/ \mathrm{C}_{60}-1$ and $\mathrm{C}_{60}-2 /$ pentacene will produce a response competition. In the thick $\mathrm{C}_{60}$ circumstance, the visible-light-triggered photogenerated carriers of $\mathrm{C}_{60}-2 /$ pentacene can hardly transfer into graphene because of depletion over the long distance, leading to a positive photocurrent in the preliminary response before infrared modulation (Fig. 3a). Infrared irradiation can induce P-doping of the graphene channel from the graphene $/ \mathrm{C}_{60}-1$ interface. Therefore, the enhanced P-doping graphene owing to infrared light modulation promotes a built-in electric field for electron injection into graphene, and $\mathrm{C}_{60}-2 /$ pentacene will dominate the photoresponse, resulting in a negative photocurrent (Fig. 3a), which offers the chance to realize logical signal reversal. It is worth noting that the power of the signal light should be compatible with that of the modulation light, and more sign reversals of photocurrents for various modulation light power densities are shown in Fig. S3. The photocurrent reversal magnitude is also related to the modulation light power density. Hence, the competition is ascribed to the different interfaces, and the asymmetric carrier transfer path provides control of the dominant interface ${ }^{44}$. The infrared light modulation can regulate this competition and neutralize the thickness depletion, which is equivalent to the thin $C_{60}$ device condition. This striking difference reveals electric field changes in the multilayer heterojunction system.

To further verify the inference proposed above, we explore power-dependent photocurrent measurements in two control groups without light modulation. As we know, there is no photocurrent reversal as a function of the input light power in most photoconductive devices. However, obviously distinct reversals of the time-resolved photocurrents between the devices with $5 \mathrm{~nm}$ and $11 \mathrm{~nm}$ thick $C_{60}$ intermediate transport layers with signal light power variation are observed (Fig. 3d). From $133.7 \mathrm{~mW} /$ $\mathrm{cm}^{2}$ to $7.7 \mu \mathrm{W} / \mathrm{cm}^{2}$ light, the negative photocurrents imply that photogenerated electrons can effectively enter graphene owing to the $\mathrm{C}_{60}-2$ /pentacene band alignment and proper thickness of intermediate transport layer $\mathrm{C}_{60}$ (Fig. 3d bottom panel), which accounts for codirectional photocurrents in light modulation (Fig. 2a). In contrast to the all negative photocurrents in the $5 \mathrm{~nm} C_{60}$ control device, we observe a two-times photocurrent reversal phenomenon in the $11 \mathrm{~nm} C_{60}$ device with decreasing power density (from $133.7 \mathrm{~mW} / \mathrm{cm}^{2}$ to $7.7 \mu \mathrm{W} / \mathrm{cm}^{2}$ ) of the input $650 \mathrm{~nm}$ light (Fig. 3d top panel). As we can see, $650 \mathrm{~nm}$ illumination with a power density exceeding $864.2 \mu \mathrm{W} / \mathrm{cm}^{2}$ of the device (Fig. 3d top panel from the dark blue to black lines) results in positive photocurrents. These positive photocurrents are the combined result of two major parts of the response: (1) the photothermoelectric part and (2) photoelectric part. As deduced from this phenomenon, the thick $C_{60}$ tends to contribute photothermoelectric currents, especially when we apply relatively high-power light. Under $650 \mathrm{~nm}$ light irradiation, the photogenerated excitons segregate and drift with the $\mathrm{C}_{60}-2 /$ pentacene band alignment. Then, the holes trapped in pentacene and a few electrons transfer into graphene owing to the depletion through $11 \mathrm{~nm} \mathrm{C}_{60}$ (Fig. 3b), which is responsible for the relatively small negative response arising from the photoelectric part 


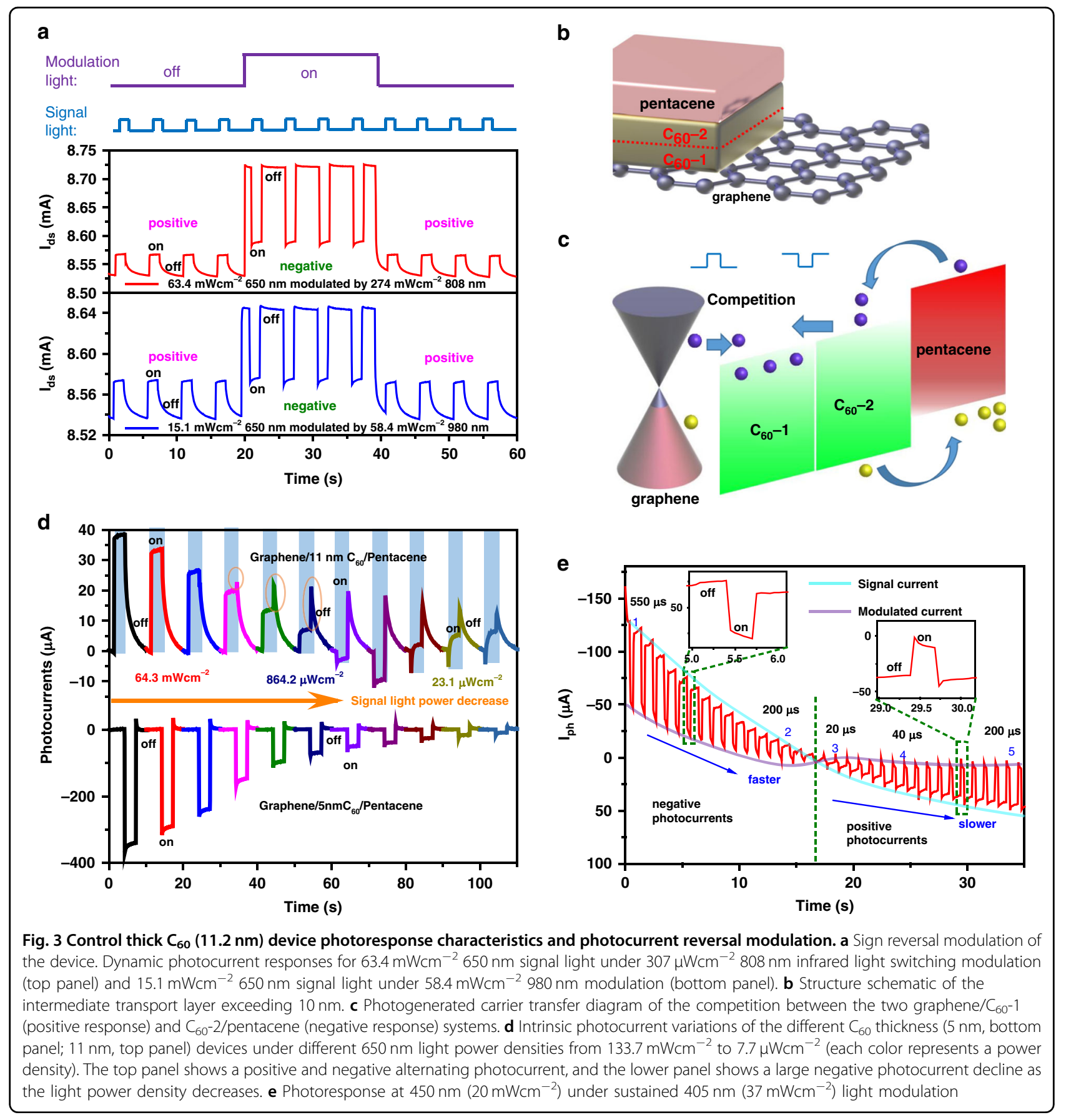

(from purple to dark purple lines in Fig. 3d top panel). We can even distinguish a pure negative response with an instantaneous sharp peak at the brink of the on-off switching moment (when the input power density is < $251.7 \mu \mathrm{W} / \mathrm{cm}^{2}$ in Fig. 3d top panel). Thus, there is still a negative response component in positive photocurrents (circled in Fig. 3d top panel). However, these sharp peaks decrease with increasing light power (from orange to dark blue lines in Fig. 3d top panel) and are ultimately submerged in the primary positive responses (from black to blue lines in Fig. 3d top panel) due to the saturation effect of photoelectric currents. By contrast, positive photocurrents exhibit remarkable growth in the corresponding power density range. Therefore, as the input light power increases, positive photothermoelectric currents will predominate in the response, causing a screening effect for the negative response. With degeneration of the photothermoelectric effect (when the $650 \mathrm{~nm}$ illumination power decreases to $\left.251.7 \mu \mathrm{W} / \mathrm{cm}^{2}\right)$, the built-in field of $\mathrm{C}_{60}$-2/pentacene leads to apparent electron injection 
(including drift and diffusion processes) into graphene, resulting in pure negative photocurrents (from violet to faint brown lines in Fig. 3d top panel). With the continuous reduction in the input light power, the concentration of electrons input into the graphene channel decreases to a low level, and few electrons can undergo the depletion or diffusion process through $\mathrm{C}_{60}-1$ and $\mathrm{C}_{60}-2$ (input power $=37.5 \mu \mathrm{W} / \mathrm{cm}^{2}$ ). Therefore, the negative photocurrents from $\mathrm{C}_{60}-2$ /pentacene vanish, leaving only the sharp peaks (Fig. $3 \mathrm{~d}$ ), and the graphene $/ \mathrm{C}_{60}-1$ interface boundary may dominate the photoresponse, leading to the positive photocurrents rising in magnitude. This fundamental powerdependent multiple state phenomenon in the photocurrent provides the possibility for photocurrent switching by light modulation.
We observed an intensive and slow negative drift in the variation in the photoconductance when stimulating the device by ultraviolet illumination without light modulation (the $405 \mathrm{~nm}$ intrinsic response depicted in Fig. S4). The complete positive and negative transition process under sustained $405 \mathrm{~nm}$ light modulation is presented in Fig. 3e. This figure shows that the photocurrent declines from negative $78.8 \mu \mathrm{A}$ to 0 and rises from 0 to positive $36.3 \mu \mathrm{A}$, with the response time changing. This gradually varied negative response offers another sustained light modulation method. Similar to electrical gate modulation, this type of sustained $405 \mathrm{~nm}$ light modulation causes an $\mathrm{N}$ type doping drift in the photocurrent measurements. Utilizing the power-dependent multiple states of the photocurrent caused by thick $\mathrm{C}_{60}$, this consecutive $\mathrm{N}$-type doping can be used to easily tune intrinsic P-type graphene
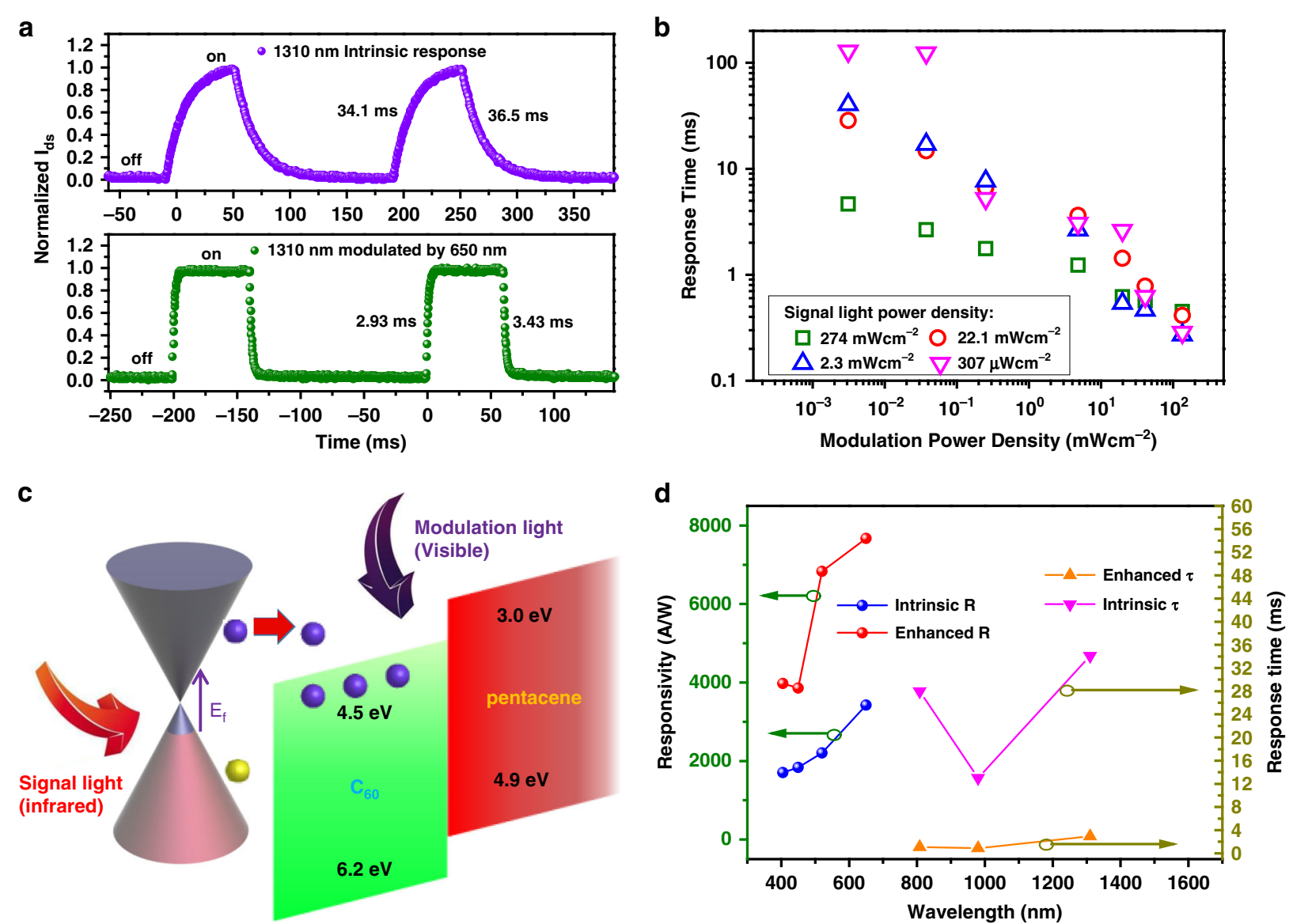

Fig. 4 Photoelectric characterization of the infrared response under visible light switching modulation ( $5 \mathrm{~nm}$ thick $\mathrm{C}_{60}$ device). a Timedependent photoresponse of the intrinsic infrared $1310 \mathrm{~nm}\left(20 \mathrm{mWcm}^{-2}\right)$ positive response and $1310 \mathrm{~nm}$ response under $650 \mathrm{~nm} 20 \mathrm{mWcm}$ light modulation measured by an oscilloscope. $\mathbf{b}$ Analysis of the response time of graphene/ $C_{60} /$ pentacene devices at different signal $(808 \mathrm{~nm})$ and modulation $(650 \mathrm{~nm})$ light power densities, $V_{\mathrm{ds}}=1 \mathrm{~V}$. c Schematic diagram of infrared light $(808-1550 \mathrm{~nm})$ working as signal light under the visiblelight-modulation-induced carrier transfer process; the small red arrow represents signal light-induced charge transfer. $\mathbf{d}$ Summary of the enhancement in the device performance of the bilayer/graphene heterojunction phototransistor in terms of the photoresponse and response time by light modulation (left panel modulation: signal light power density: $28.3 \mu \mathrm{Wcm} \mathrm{cm}^{-2}(405 \mathrm{~nm}), 17.4 \mu \mathrm{Wcm}^{-2}(450 \mathrm{~nm}), 36.7 \mu \mathrm{Wcm}{ }^{-2}(520 \mathrm{~nm})$ $23.4 \mu \mathrm{Wcm}^{-2}(650 \mathrm{~nm})$, and modulation light power density of nearly $300 \mu \mathrm{Wcm}^{-2}$; right panel modulation: signal and modulation light power densities of $\sim 20 \mathrm{mWcm}^{-2}$ ) 
into N-type. The N-type doping drift of graphene o to ultraviolet light is attributed to oxygen chemisorption, and the ultraviolet light removes the chemisorbed oxygen on the surface of graphene. At present, the electrons from $\mathrm{C}_{60}$ /pentacene transfer into $\mathrm{N}$-type graphene, leading to positive photocurrents. We captured several transient response times measured by an oscilloscope (as shown in Fig. S5), which indicates a photoresponsivity-response time trade-off. The intrinsic response time can be modulated to as low as $20 \mu \mathrm{s}$ for a weak positive photoresponse, as depicted in Fig. 3e. The intrinsic rise time (or response time) ranges from 20 to $550 \mu \mathrm{s}$, showing the $\mathrm{\tau}$-light modulation relationship in our device. The similar relationship can enable a parallel comparison with the $\tau-V_{\mathrm{g}}$ relationship in the graphene/perylenetetracarboxylic dianhydride/pentacene device ${ }^{24}$. The relatively fast operation time $(20 \mu \mathrm{s})$ at the cost of the photocurrent shows highspeed application potential in light modulation devices. Furthermore, the dynamic light-modulated photoresponsivity and response time suggest a thicknessdependent carrier transfer feature and potential for replacement of electrical modulation.

Finally, we carefully investigate the infrared response photocurrent variation with visible light modulation. Figure 4a plots the time-resolved variation characteristics of the infrared response, with a faster response time under visible light modulation. At the expense of the photocurrent, the rise and recovery times of the positive $1310 \mathrm{~nm}$ response (power density $=20 \mathrm{~mW} / \mathrm{cm}^{2}$ ) can be reduced from 34.1 and $36.5 \mathrm{~ms}$ to 2.93 and $3.43 \mathrm{~ms}$ under $650 \mathrm{~nm}$ illumination with a power of $20 \mathrm{~mW} / \mathrm{cm}^{2}$ at the cost of a low gain (G). As the variation in the Fermi level is associated with the power density of the modulation light, the distinct doping levels of graphene affect the band bending and thus the transmission, which is closely related to the response time. Therefore, we present the power-dependent response time curves in Fig. 4b, which indicate an operable tuneable bandwidth from 10 to $3 \times$ $10^{3} \mathrm{~Hz}$. All curves demonstrate an improved response time as the modulation light power density increases, and the response time can be promoted $\sim 10^{3}$ times when $307 \mu \mathrm{W} / \mathrm{cm}^{2} 808 \mathrm{~nm}$ signal light is modulated by $650 \mathrm{~nm}$ light with a power of $133.7 \mathrm{~mW} / \mathrm{cm}^{2}$. Distinct from the visible response modulated by infrared light, we found complete inhibition of the photocurrent in different light modulation bands (Fig. S6a, b). The $\mathrm{C}_{60}$ /pentacene bilayer generates the majority of electrons injected into graphene, resulting in $\mathrm{N}$-type doping under visible light illumination. The intrinsic positive photocurrents can be modulated to produce smaller photocurrents owing to the builtin electric field of graphene $/ \mathrm{C}_{60}$ diminishing as graphene $\mathrm{N}$-type doping is enhanced (see Fig. 4c). To obtain more details of the visible light modulation, we also perform photocurrent dynamic measurements under different incident modulation light power densities (see Fig. S6c); as the modulation light power density decreases, all curves shows a decline in the photocurrent modulation result. Compared with the infrared light modulation, no direction variation but a decline in the photocurrent is observed even at low power densities of the signal and modulation light (as shown in Fig. S7).

\section{Discussion}

The light modulation method mentioned above involves mutual modulation between two light wavelength regimes (except for the special circumstance of $405 \mathrm{~nm}$ modulation with removal of chemisorbed oxygen). Therefore, this graphene $/ \mathrm{C}_{60} /$ pentacene structure splits the detection range into two regions, $405-650$ and $808-1550 \mathrm{~nm}$, owing to the bidirectional response. The upper layer material determines the positive and negative response transition points, which can affect the distinct light modulation regions. To exclude some accidental factors, the photoresponse characteristics when the dynamics are modulated in one region (both the signal and modulation light are in one region) are investigated in Fig. S8, which exhibits a normal saturation relation between them. Different from the traditional hybrid modulation phototransistor using one gate light or source light beam to tune signals, our all-light-input phototransistor with a tuneable dynamic response feature under light modulation demonstrates that light modulation can replace most of the function of electrical modulation by a gate and even trigger multiple state competition in the vertical direction. The photocurrent polarity variation phenomenon with the substitution of electrical modulation paves the way for developing polarity-controlled all-optical-input devices ${ }^{7}$.

The high photoresponsivity and low response time of the $5 \mathrm{~nm} \mathrm{C}_{60} / 10 \mathrm{~nm}$ pentacene device can be achieved through our two distinct light modulations, where the two important parameters are enhanced in different situations, as summarized in Fig. 4d (the left panel shows modulation by infrared light, and the right panel shows modulation by visible light). The promotion of photocurrents via suitable infrared modulation light power ensures efficient transport of carriers and avoids a saturation effect at the cost of the recovery time in the visible region. The visible light $\mathrm{N}$-doping modulation improves the response and recovery times at the sacrifice of the photocurrent. Moreover, the asymmetric amplitude and response time of the logical photocurrent reversals may affect back-end signal acquisition. However, it is advantageous that infrared modulation light can act as a switch to turn on or off the logical signal reversal function, and simultaneously, all the input signals and modulations were based on light.

In summary, we demonstrated two distinct light modulations in thin and thick $\mathrm{C}_{60}$ bilayer phototransistors 
(graphene $/ \mathrm{C}_{60} /$ pentacene). The thick $\mathrm{C}_{60}$ layer device exhibits a more distinct modulation phenomenon as the thickness of the intermediate transport layer increases, realizing logical switching via light modulation, which shows great potential for fabricating optical polarity modulation devices. Continuous violet illumination enables us to observe a repeatable positive-negative alternating process and an intrinsic visible response $\tau$ tuneable down to $\sim 20 \mu \mathrm{s}$. Moreover, the visible light modulation provides a tuneable bandwidth from 10 to $3 \times$ $10^{3} \mathrm{~Hz}$ for adjusting infrared photocurrents. The powerrelated tuneable photocurrent phenomenon provides a new effective light modulation method for enhanced response, where the photoresponse is increased from $3425 \mathrm{~A} / \mathrm{W}$ up to $7673 \mathrm{~A} / \mathrm{W}$. These light modulation results not only reveal the carrier transport mechanism in bidirectional phototransistors but also present the advantage and mechanism of light modulation, which provides a reliable method for future development of more innovative and functional optoelectronic devices.

\section{Materials and methods \\ Device fabrication and characterization}

Single-layer CVD graphene was transferred to an $\mathrm{n}+\mathrm{Si} /$ $\mathrm{SiO}_{2}$ substrate $\left(\mathrm{SiO}_{2} 285 \mathrm{~nm}\right)$ by a solution method. The CVD graphene was purchased from Nanjing MKNANO Technology Inc. The $5 \mathrm{~nm} \mathrm{C}_{60}\left(\right.$ at $\left.430{ }^{\circ} \mathrm{C}\right)$ deposition rate was $0.02 \mathrm{~nm} / \mathrm{s}$ (vacuum $=10^{-5}$ Torr). Then, $10 \mathrm{~nm}$ thick pentacene (at $155^{\circ} \mathrm{C}$ ) was vapor-deposited onto $5 \mathrm{~nm} \mathrm{C} \mathrm{C}_{60}$ at a starting deposition rate of $0.02 \mathrm{~nm} / \mathrm{s}$, which was gradually increased to $0.04 \mathrm{~nm} / \mathrm{s}$. All film thicknesses mentioned above were determined by a SQC-310 deposition controller. The pentacene material, rated at over 98\% purity, was purchased from MREDA Technology Inc. made in the USA. The $\mathrm{C}_{60}$ material, rated at over 99.5\% purity, was purchased from Polymer Xi'an Baotelai Light Technology Inc.

\section{Light-modulation measurement methods}

We used a response laser beam and a modulation laser beam to investigate light modulation characteristics. The transfer characteristics and light modulation optoelectronic characteristics data were measured by a Keithley 2636b source meter analyzer and a PDA FS-Pro semiconductor analyzer with a probe station. Highly timeresolved photocurrent signals under light modulation were obtained by an oscilloscope with a pulse source, which were amplified and detected using the current amplifier (Stanford SR570) technique.

The absorption data of the graphene $/ \mathrm{C}_{60} /$ pentacene and $\mathrm{C}_{60}$ /pentacene control groups were collected by a UV2600, and Raman spectra measurements were performed by a confocal microprobe Raman spectrometer (RENISHAW inVia Raman Microscope) under the illumination of a $514 \mathrm{~nm}$ helium-neon laser with a power of $2 \mathrm{~mW}$.

\begin{abstract}
Acknowledgements
This work was supported in part by the National Natural Science Foundation of China (no. 61922022, 61421002, 61875031, 61734003, 61674157, 11734016), the Opened Fund of the State Key Laboratory of Integrated Optoelectronics (no. IOSKL2017KF17), and the Key Research Project of Frontier Science of CAS (grant no. QYZDB-SSW-JSC031).
\end{abstract}

\section{Author details}

${ }^{1}$ School of Optoelectronic Science and Engineering, University of Electronic Science and Technology of China, Chengdu 610054, China. ${ }^{2}$ State Key Laboratory of Infrared Physics, Shanghai Institute of Technical Physics, Chinese Academy of Science, 500 Yutian Road, Shanghai 200083, China. ${ }^{3}$ Hangzhou Institute for Advanced Study, University of Chinese Academy of Sciences, Hangzhou 310024, China. ${ }^{4}$ Henan Key Laboratory of Diamond Optoelectronic Materials and Devices, School of Physics and Engineering, Zhengzhou University, Zhengzhou 450001, China. ${ }^{5}$ National Laboratory of Solid State Microstructures, School of Electronic Science and Engineering, and Collaborative Innovation Center of Advanced Microstructures, Nanjing University, Nanjing 210093, China. ${ }^{6}$ State Key Laboratory of Electronic Thin Films and Integrated Devices, University of Electronic Science and Technology of China, Chengdu 610054, China

\section{Author contributions}

$\mathrm{JW}, \mathrm{XW}, \mathrm{WH}$ and $\mathrm{JH}$ conceived and supervised the project. JH prepared the device and performed the photocurrent measurements and characterizations. $\mathrm{JH}$ and $\mathrm{QH}$ set up the experimental platform, and $\mathrm{JH}, \mathrm{MY}, \mathrm{WH}, \mathrm{FZ}, \mathrm{MX}, \mathrm{FM}, \mathrm{CS}$, $Y Z, J G, Z W, X C, Q L$, and $H Z$ performed the data analysis. JW and JH co-wrote this paper. All authors discussed the results and commented on the manuscript.

\section{Conflict of interest}

The authors declare that they have no conflict of interest.

Supplementary information is available for this paper at https://doi.org/ 10.1038/s41377-020-00406-4.

Received: 9 May 2020 Revised: 31 August 2020 Accepted: 1 September 2020

Published online: 23 September 2020

\section{References}

1. Meric, I. et al. Current saturation in zero-bandgap, top-gated graphene fieldeffect transistors. Nat. Nanotechnol. 3, 654-659 (2008).

2. $\mathrm{Ma}$, Q. et al. Giant intrinsic photoresponse in pristine graphene. Nat. Nanotechnol. 14, 145-150 (2019).

3. Akinwande, D. et al. Graphene and two-dimensional materials for silicon technology. Nature 573, 507-518 (2019).

4. Li, Q. T. et al. Transparent multispectral photodetectors mimicking the human visual system. Nat. Commun. 10, 4982 (2019).

5. Lien, M. B. et al. Ranging and light field imaging with transparent photodetectors. Nat. Photonics 14, 143-148 (2020).

6. Guo, J. S. et al. High-performance silicon-graphene hybrid plasmonic waveguide photodetectors beyond 1.55 um. Light: Sci. Appl. 9, 29 (2020).

7. Chen, J. H. et al. An all-optical modulator based on a stereo graphene-microfiber structure. Light: Sci. Appl. 4, e360 (2015).

8. He, X. Y. et al. Investigation of phonon scattering on the tunable mechanisms of terahertz graphene metamaterials. Nanomaterials 10, 39 (2020).

9. He, X. Y. et al. Tunable strontium titanate terahertz all-dielectric metamaterials. J. Phys. D. Appl. Phys. 53, 155105 (2020).

10. Nair, R. R. et al. Fine structure constant defines visual transparency of graphene. Science 320, 1308 (2008).

11. Pan, R. et al. High-responsivity photodetectors based on formamidinium lead halide perovskite quantum dot-graphene hybrid. Part. Part. Syst. Charact. 35, 1700304 (2018). 
12. Konstantatos, G. et al. Hybrid graphene-quantum dot phototransistors with ultrahigh gain. Nat. Nanotechnol. 7, 363-368 (2012).

13. Long, M. S. et al. Broadband photovoltaic detectors based on an atomically thin heterostructure. Nano Lett. 16, 2254-2259 (2016).

14. Yu, W. J. et al. Highly efficient gate-tunable photocurrent generation in vertical heterostructures of layered materials. Nat. Nanotechnol. 8 952-958 (2013).

15. Yang, T. F. et al. Van der Waals epitaxial growth and optoelectronics of large-scale $\mathrm{WSe}_{2} / \mathrm{SnS}_{2}$ vertical bilayer $\mathrm{p}-\mathrm{n}$ junctions. Nat. Commun. 8, 1906 (2017).

16. Wu, F. et al. High efficiency and fast van der Waals hetero-photodiodes with a unilateral depletion region. Nat. Commun. 10, 4663 (2019).

17. Wang, G. C. et al. Interlayer coupling induced infrared response in $\mathrm{WS}_{2} / \mathrm{MoS}_{2}$ heterostructures enhanced by surface Plasmon resonance. Adv. Funct. Mater. 28, 1800339 (2018).

18. Bhuiyan, M. A. et al. Photoquantum hall effect and light-induced charge transfer at the interface of graphene/InSe heterostructures. Adv. Funct. Mater. 29, 1805491 (2019).

19. Qin, S. C. et al. Planar graphene- $C_{60}$-graphene heterostructures for sensitive UV-Visible photodetection. Carbon 146, 486-490 (2019).

20. Deng, J. N. et al. $\mathrm{MoS}_{2} / \mathrm{HfO}_{2} /$ silicon-on-insulator dual-photogating transistor with ambipolar photoresponsivity for high-resolution light wavelength detection. Adv. Funct. Mater. 29, 1906242 (2019).

21. Muench, J. E. et al. Waveguide-integrated, plasmonic enhanced graphene photodetectors. Nano Lett. 19, 7632-7644 (2019).

22. Flöry, N. et al. Waveguide-integrated van der Waals heterostructure photodetector at telecom wavelengths with high speed and high responsivity. Nat. Nanotechnol. 15, 118-124 (2020)

23. Han, J. Y. et al. Graphene/organic semiconductor heterojunction phototransistors with broadband and bi-directional photoresponse. Adv. Mater. 30, 1804020 (2018).

24. Chen, X. Q. et al. Improving the performance of graphene phototransistors using a heterostructure as the light-absorbing layer. Nano Lett. 17, 6391-6396 (2017).

25. Cakmakyapan, S. et al. Gold-patched graphene nano-stripes for highresponsivity and ultrafast photodetection from the visible to infrared regime. Light: Sci. Appl. 7, 20 (2018).

26. Gao, L. et al. Broadband, sensitive and spectrally distinctive $\mathrm{SnS}_{2}$ nanosheet/ PbS colloidal quantum dot hybrid photodetector. Light: Sci. Appl. 5, e16126 (2016).
27. Wang, Y. et al. Negative photoconductance in van der waals heterostructurebased floating gate phototransistor. ACS Nano 12, 9513-9520 (2018).

28. Gong, F. et al. High-sensitivity floating-gate phototransistors based on WS and $\mathrm{MoS}_{2}$. Adv. Funct. Mater. 26, 6084-6090 (2016).

29. Liu, X. L. et al. Organic charge-transfer interface enhanced graphene hybrid phototransistors. Org. Electron. 64, 22-26 (2019).

30. Qin, S. C. et al. Sensitive and robust ultraviolet photodetector array based on self-assembled graphene/ $C_{60}$ Hybrid films. ACS Appl. Mater. Interfaces 10, 38326-38333 (2018)

31. Wang, X. D. et al. Ultrasensitive and broadband $\mathrm{MoS}_{2}$ photodetector driven by ferroelectrics. Adv. Mater. 27, 6575-6581 (2015).

32. Xie, C. et al. Ultrasensitive broadband phototransistors based on perovskite/ organic-semiconductor vertical heterojunctions. Light: Sci. Appl. 6, e17023 (2017).

33. Cao, M. et al. Enhanced photoelectrical response of thermodynamically epitaxial organic crystals at the two-dimensional limit. Nat. Commun. 10, 756 (2019).

34. Fang, H. H. et al. Visible light-assisted high-performance mid-infrared photodetectors based on single InAs nanowire. Nano Lett. 16, 6416-6424 (2016).

35. Gao, X. D. et al. All-optical-input transistors: light-controlled enhancement of Plasmon-induced photocurrent. Adv. Funct. Mater. 28, 1802288 (2018).

36. Lee, J., Jadhav, P. \& Baldo, M. A. High efficiency organic multilayer photodetectors based on singlet exciton fission. Appl. Phys. Lett. 95, 033301 (2009).

37. Kang, S. J. et al. Energy level diagrams of $C_{60} /$ pentacene/Au and pentacene/ C60/Au. Synth. Met. 156, 32-37 (2006).

38. Kim, K. et al. Structural and electrical investigation of $\mathrm{C}_{60}$-graphene vertical heterostructures. ACS Nano 29, 5922-5928 (2015).

39. Liu, X. L. et al. Epitaxial ultrathin organic crystals on graphene for highefficiency phototransistors. Adv. Mater. 28, 5200-5205 (2016).

40. Shokouh, S. H. H. et al. Molybdenum disulfide nanoflake-zinc oxide nanowire hybrid photoinverter. ACS Nano 8, 5174-5181 (2014).

41. Kim, S. et al. Light sensing in a photoresponsive, organic-based complementary inverter. ACS Appl. Mater. Interfaces 3, 1451-1456 (2011).

42. Gao, L. et al. Defect-engineered atomically thin $\mathrm{MoS}_{2}$ homogeneous electronics for logic inverters. Adv. Mater. 32, 1906646 (2020).

43. Jeong, J. W. et al. Tunnelling-based ternary metal-oxide-semiconductor technology. Nat. Electron. 2, 307-312 (2019).

44. Chen, J. J. et al. Photovoltaic effect and evidence of carrier multiplication in graphene vertical homojunctions with asymmetrical metal contacts. ACS Nano 9, 8851-8858 (2015). 